a

Budding yeast

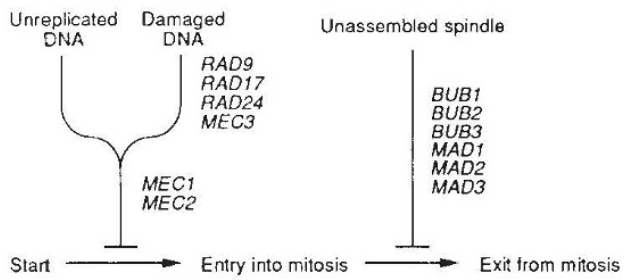

$b$

Fission yeast



FIG. 3 Feedback controls over mitosis in the budding and fission yeasts. a, The genes involved in feedback control over the entry into and exit from mitosis in budding yeast. $b$, The genes involved in feedback control over the entry into mitosis in fission yeast.
DNA (T. Weinert, personal communication), suggesting that, although different sensors detect damaged and unreplicated DNA, both feedback controls converge to act on the same checkpoint in the cell-cycle engine (Fig. $3 a$ ). The nature of the lesions in DNA that are detected as damaged or unreplicated DNA is not known.

Deletion of the RAD9 gene increases the rate of chromosome loss 20 -fold, but is not lethal, suggesting that the feedback control that detects damaged DNA is not required in every cell cycle $^{18}$. Although this control has been investigated using experimentally induced DNA damage, cells also produce molecules that are seen as damaged DNA during the course of normal metabolism, such as the single-stranded nicks and gaps produced during DNA replication. The simplest interpretation of the viability of cells lacking $R A D 9$ is that cells use relative timing and feedback controls as redundant ways of solving the completion problem. Even in nonembryonic cells, the relative timing of downstream events and the cell-cycle engine guarantees that in most cells the downstream events are completed before the cell-cycle engine makes its next transition. Therefore if the feedback control has been inactivated, relative timing ensures that most cells complete downstream events before the next cell-cycle transition is induced. Similarly feedback controls prevent those cells where downstream events have been delayed, either stochastically or by experimental or environmental insults, from incurring death and genetic damage. If this analysis is correct, the feedback controls do not actively restrain the cellcycle engine in most cycles, because relative timing ensures that on average the downstream events are completed before the cell reaches the checkpoint. In support of this argument, deletion of most ${ }^{18,19}$, but not all ${ }^{20}$, feedback control genes allow populations of cells to grow, albeit with increased frequencies of cell death and genetic damage. The level of redundancy between different feedback controls and relative timing is an important unresolved question.

The response to DNA damage has also been investigated in the fission yeast (Fig. $3 b$ ). Mutations in a number of rad genes allow cells with damaged or unreplicated DNA to enter mitosis ${ }^{21,22}$. Other mutations allow cells with unreplicated DNA to enter mitosis, but do not affect the ability of damaged DNA to arrest the cell cycle ${ }^{23}$. The relationship between the genes involved in budding and fission yeast feedback controls is not known.

A temperature-sensitive mutation (inactive at higher temperature) in the mammalian RCC1 (repressor of chromosome condensation) gene causes cultured hamster cells that are shifted-up to the nonpermissive temperature during $\mathrm{S}$ phase to cease DNA replication and enter mitosis prematurely. Cells shifted up early in G1 do not synthesize any DNA after shift-up and do not enter mitosis ${ }^{24}$. This mutation clearly affects both

\title{
BOX 1 A cell cycle refresher
}

THE past five years have seen a prodigious advance in our understanding of the cell cycle, which is very briefly reviewed here. The key component proteins of the cell-cycle engine are members of the p34 family and cyclins. The protein p $34^{c 062}$ was identified as the product of two cell division cycle genes, cdc2 of the fission yeast, Schizosaccharomyces pombe, and $\mathrm{CDC} 28$ of the budding yeast, Saccharomyces cerevisiae. Cyclins were discovered by studying patterns of protein synthesis in fertilized sea urchin eggs. The stability of cyclin proteins varies throughout the cell cycle, whereas $p 34^{\text {cdo2 }}$ is stable. Cyclins are divided into a number of classes based on sequence similarity and physiological function: mitotic cyclins (cyclin B), S-phase cyclins (cyclin A) and G1 cyclins (called $\mathrm{Cln}$ in yeast and cyclins $C, D, E$ and $F$ in vertebrates).

Figure 1 a shows the cell cycle of a yeast cell. In G1, the beginning of the cell cycle, p34 ${ }^{\text {coc2 }}$ lacks any associated cyclins and has no protein kinase activity. As long as cells are adequately supplied with nutrients and sufficiently large, they accumulate G1 cyclins that bind to p34 $4^{\operatorname{coc} 2}$ and activate its protein kinase activity, thereby inducing cells to pass through Start, the first transition in the cell cycle. The Start-promoting activity of p34 ${ }^{\circ 002}$ induces cells to replicate their DNA (entering S phase) and their microtubule organizing centre (MTOC). In yeasts the molecular regulation of Start is fairly well understood, whereas our understanding of how mammalian cells pass the restriction point (the equivalent of Start) is much more fragmentary. In mammalian cells and budding yeast the restriction point/Start is the principal point in the cell cycle where proliferation is regulated by nutrient availability and growth factors (Fig. 1b).

After passing Start, cells begin to synthesize cyclin B which accumulates and binds to $p 34^{c o c 2}$. This complex is inactive because the binding of cyclin B to p34 ${ }^{c d c 2}$ induces the phosphorylation of tyrosine 15 of p34 ${ }^{\text {coc2 }}$, which inhibits its protein kinase activity. The inactive complex, often referred to as preMPF, is also phosphorylated on threonine 160 . This phosphorylation is required for MPF activity but is not sufficient to overcome the inhibitory effect of tyrosine phosphorylation. The subsequent removal of the tyrosine phosphate from $p 34^{\text {coc2 }}$ during late $\mathrm{G} 2$ activates MPF and leads to the induction of mitosis ( $M$ phase). These post-translational reactions play a major role in regulating the timing of MPF activation and are subject to complex physiological regulation. The tyrosine kinase was initially identified as the product of the fission yeast wee1 gene, which acts as an inhibitor of entry into mitosis, whereas the phosphatase is the product of the fission yeast cdc25 gene which acts to promote entry into mitosis. Active MPF seems to activate the tyrosine phosphatase and inhibit the tyrosine kinase that modifies $p 34^{c 002}$, leading to an explosive activation of MPF that drives cells rapidly and irreversibly into mitosis. MPF induces chromosome condensation, nuclear envelope breakdown and assembly of the mitotic spindle. MPF induces nuclear envelope breakdown directly by phosphorylating the nuclear lamins, but it is not known whether chromosome condensation and spindle assembly are induced directly by MPF or indirectly by other enzymes activated by MPF.

Once MPF is active it induces the activation of enzymes that conjugate ubiquitin to cyclin and thereby target cyclin for degradation by a well chraracterized system that proteolyses poly-ubiquitinated proteins. The degradation of cyclin destroys the kinase activity of MPF and induces the cell cycle to progress into the next interphase and allows cyclin to become stable again. The degradation of cyclin and the inactivation of MPF induce chromosome segregation, chromosome decondensation, nuclear reformation and cytokinesis.

Somatic and embryonic cell cycles differ mainly in the regulation of DNA synthesis and microtubule organizing centre duplication. In embryonic cell cycles DNA and microtubule organizing centre replication are downstream events induced by the inactivation of MPF rather than by passage through Start. Both of these events occur as soon as nuclear envelopes have reformed after the inactivation of MPF, and there is no evidence for any equivalent of Start in the embryonic cell cycle. For further details and full references see refs $2-5$. 